\title{
étude numérique du tassement d'une fondation de grand diamètre sur argile surconsolidée
}

\author{
numerical study of the settlement of a large diameter \\ foundation on overconsolidated clay
}

\author{
A. PECKER \\ Gérant, Géodynamique et Structure * \\ F. ROPERS \\ Ingénieur, Mécasol SA*" \\ P. BOZETTO \\ EDF SEPTEN $* \cdots$
}

Rev. Franç. Géotech. n 47 , p.p. 17-28 (avril 1989)

\section{Résumé}

EDF a été confronté aux problèmes de prévision des tassements sous les ouvrages de centrales nucléaires. Pour apprécier la validité des méthodes usuelles de calcul (œdométrique et pressiométrique), appliquées au tassement d'une fondation de grand diamètre reposant sur une couche homogène d'argile surconsolidée, le SEPTEN a eu recours à la simulation numérique.

Le sol est modélisé par une loi élastoplastique dont les paramètres ont été déterminés à partir d'essais sur échantillons intacts d'argile du Gault. La simulation d'essais œdométriques et pressiométriques et leur comparaison aux essais réels ont validé la loi.

L'évaluation numérique du tassement d'une fondation rigide de grand diamètre a été comparée à l'estimation déduite d'essais œdométriques ou pressiométriques. L'étude a montré la fiabilité de la méthode œdométrique dans ce cas, sous réserve d'essais de bonne qualité. La méthode pressiométrique dépend fortement du coefficient $\alpha$, qui devrait être légèrement supérieur à 1 dans le cas du matériau étudié, ce qui est en dehors des normes pressiométriques.

\begin{abstract}
EDF was faced with the problems of forecasting settlements under nuclear power plants. To ascertain the validity of the currently used methods (from cedometer tests and pressuremeter tests) of evaluating settlement of a large diameter foundation on an homogeneous layer of overconsolidated clay, SEPTEN has opted for numerical simulation.

An elastoplastic constitutive model was used for the soil. The model parameters were determined from tests performed on undisturbed samples of the Gault clay. Simulation of œdometer and pressumeter tests compared to actual tests has validated the model.

The numerical evaluation of the settlement of a large diameter foundation was compared to that deduced from œdometer and pressuremeter tests. The study has shown the good reliability of the œdometric method, provided samples are of high quality. The pressuremeter test method has proved to be strongly dependent on the coefficient $\alpha$, necessarily greater than 1 for the Gault clay.
\end{abstract}

\footnotetext{
- 6, rue Eugène-Oudiné, 75013 Paris

* 6, rue Eugène-Oudiné, 75013 Paris

*.. 12-14, avenue Dutriévoz, 69628 Villeurbanne Cedex.
} 


\section{INTRODUCTION}

A l'occasion de la construction des centrales nucléaires, EDF a été confronté aux problèmes de la prévision des tassements sous les ouvrages. En effet, les bâtiments des ilôts nucléaires transmettent au sol des pressions comprises entre 0,25 et $0,50 \mathrm{MPa}$; ces variations de charges entre bâtiments sont à l'origine de tassements différentiels dont il convient d'estimer l'amplitude avant construction, pour prévoir en conséquence les tuyauteries de liaison, les joints, etc.

Par ailleurs, les réfrigérants atmosphériques constituent des structures pour lesquelles les effets de tassements différentiels sont particulièrement préjudiciables.

La méthode de prévision des tassements la plus utilisée pour les ouvrages des sites nucléaires est basée sur l'interprétation des résultats d'essais œedométriques effectués sur les échantillons prélevés au cours de la campagne de reconnaissance.

Assez récemment, le pressiomètre a été utilisé en matière de prévisions de tassements, l'interprétation des essais in situ faisant alors appel aux formules empiriques classiques.

Les deux méthodes ont conduit à des estimations franchement divergentes sur certains sites sans qu'il soit possible de connaître précisément l'origine de ces divergences:

- remaniement des échantillons dits intacts;

- représentativité de l'échantillon ou de l'essai in situ ;

- fiabilité des corrélations pour l'essai pressiométrique.

Face à cette situation, le SEPTEN a estimé que la simulation numérique du tassement d'une fondation de grand diamètre reposant sur un site idéal constitué d'argile fortement surconsolidée homogène pouvait permettre de juger de la validité des deux méthodes de calcul usuellement employées. Cette simulation numérique présente l'avantage, par rapport à une expérimentation en vraie grandeur d'être moins coûteuse et moins délicate d'interprétation et de s'affranchir du problème de remaniement des échantillons lors du prélèvement ; en contre-partie, elle exige la connaissance d'une loi de comportement réaliste pour le sol.

La démarche mise en cuvre pour cette étude est la suivante :

- détermination des paramètres de la loi de comportement de sol à partir d'essais réalisés sur des échantillons intacts ;

- validation de la loi de comportement par simulation numérique d'essais œdométriques et d'essais pressiométriques et comparaison aux essais réels ;

- évaluation numérique du tassement d'une fondation représentative d'un bâtiment réacteur reposant sur une épaisseur homogène de sol. Cette évaluation a été comparée à l'estimation qui aurait pu être faite à partir des essais pressiométriques et œedométriques obtenus par simulation numérique.

Dans la suite de l'article, on aborde successivement les points suivants:
- choix du matériau ;

- choix du modèle de comportement ;

- détermination des paramètres de la loi de comportement ;

- validation du modèle de comportement ;

- simulation numérique des essais œedométriques et pressiométriques ;

- évaluation numérique du tassement de la fondation.

\section{CHOIX DU MATÉRIAU}

Le matériau retenu pour l'étude est l'argile du Gault rencontrée sur le site de la centrale nucléaire de Penly. Ce matériau a été choisi car il a fait l'objet d'une campagne de reconnaissance particulièrement soignée permettant l'obtention d'échantillons de très grande qualité.

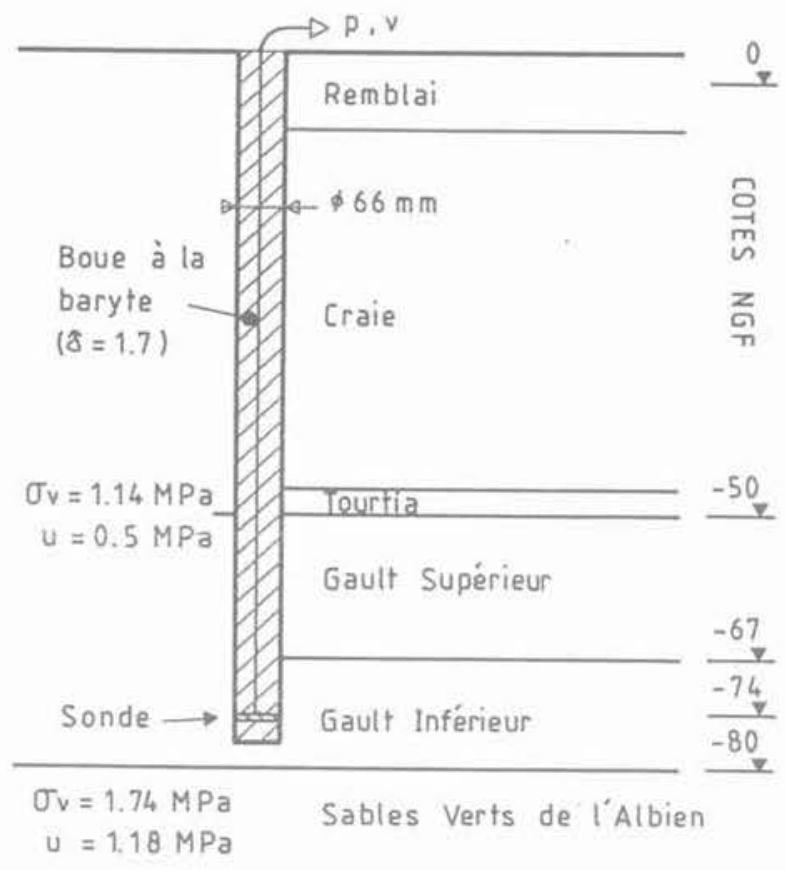

Fig. 1. - Site de Penly - Stratigraphie.

Fig. 1. - Penly site - Soil profile

La coupe stratigraphique du site est donnée sur la figure 1. Le sol est constitué depuis la surface d'une couche de remblai surmontant la craie sur environ $45 \mathrm{~m}$ d'épaisseur. Sous la craie, on rencontre l'argile du Gault dans laquelle deux niveaux peuvent être distingués : le Gault dit supérieur, fortement carbonaté et fissuré et le Gault inférieur, plus homogène. L'argile du Gault surmonte la couche des sables verts de l'Albien qui sur le site de Penly, est le siège d'une nappe fortement artésienne (surpression par rapport au niveau hydrostatique de l'ordre de $0,4 \mathrm{MPa}$ ). La présence de cette nappe a nécessité la réalisation de forages avec une boue à la baryte de densité 1,7. Les échantillons du Gault inférieur ont été prélevés à l'aide 
d'un double carottier, remontés à la surface, sortis de leur enveloppe, grattés pour enlever le cake de boue, paraffinés et immédiatement acheminés au laboratoire. Les éprouvettes destinées à la réalisation des essais ont été très soigneusement taillées à la main dans les carottes de diamètre $86 \mathrm{~mm}$.

D'un point de vue géotechnique, l'argile du Gault inférieur du site de Penly est une argile plastique (indice de plasticité de $40 \%$ ), fortement surconsolidée. Pour ce matériau, le coefficient $K_{0}$ de poussée des terres au repos a été estimé à 1,3. La teneur en carbonate de l'argile est faible $(<10 \%)$. Les caractéristiques de cisaillement intergranulaire du matériau sont $\phi^{\prime}=25^{\circ}, \mathrm{C}^{\prime}=0,2 \mathrm{MPa}$.

\section{MODÈLE DE COMPORTEMENT}

Le sol est caractérisé par une loi de comportement définie en contraintes effectives, de type élastoplastique avec écrouissage cinématique, utilisant le concept de surfaces de charge multiples. Cette loi a été développée par J.-H. PREVOST [7]. L'eau interstitielle est considérée comme un fluide parfait. Le couplage entre le squelette du sol et l'eau interstitielle est introduit par l'intermédiaire d'une loi de conservation qui, dans les cas les plus simples, se réduit à la loi de Darcy. Seules les caractéristiques générales de la loi de comportement seront rappelées ci-après. Les équations générales sont détaillées dans la référence précitée.

\subsection{Domaine élastique}

L'élasticité est supposée isotrope. Toute l'anisotropie de comportement est supposée reflétée par la plasticité. Les modules élastiques volumétrique et de cisaillement. $B$ et $G$, dépendent de la contrainte movenne effective $\mathrm{p}$ suivant la relation :

$$
\frac{B}{B 1}=\frac{G}{G 1}=\left[\frac{p}{p_{1}}\right]^{n}
$$

B1 et G1 sont les modules mesurés pour la contrainte de référence $\mathrm{p}_{1}, \mathrm{n}$ est un paramètre déterminé expérimentalement à l'aide d'un essai de colonne résonante par exemple.

\subsection{Surfaces de charge}

Le concept de surfaces de charge multiples, incluses les unes dans les autres, est utilisé afin de pouvoir linéariser par segments la courbe effort-déformation. A chacune de ces surfaces, est associé un module plastique constant mais dépendant de l'état de contraintes $\mathrm{p}$ suivant une relation analogue à celle donnée par l'équation 1. Les surfaces de charge sont de type Drucker-Prager. Dans l'espace des contraintes principales, ces surfaces correspondent à des cônes dont le sommet $\mathrm{A}$ est situé sur l'axe hydrostatique à une distance $C^{\prime} \operatorname{cotg} \phi^{\prime}$ de l'origine (fig. 2). La trace de la surface de charge dans un plan déviatorique $\pi$
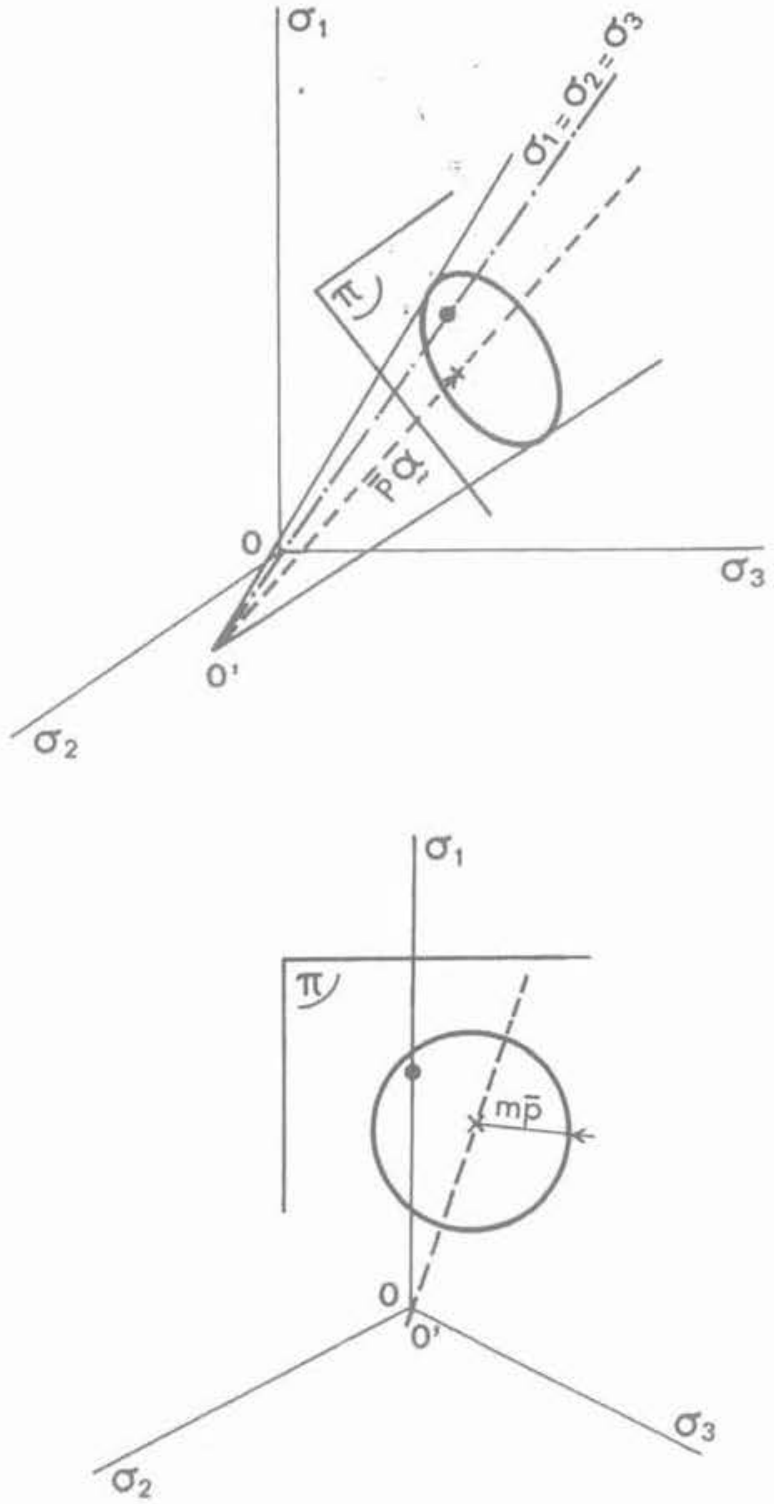

Fig. 2. - Surface de charge dans l'espace des contraintes effectives principales.

Fig. 2. - Yield surface in the principle effective stress space

$(\mathrm{p}=$ constante) est un cercle dont le centre est translaté du vecteur $\mathrm{p} \alpha$ par rapport à l'intersection de l'axe hydrostatique avec ce plan ( $\alpha$ représente un tenseur d'anisotropie du matériau). Si l'axe du cône représentant la surface de charge coïncide avec laxe hydrostatique $(\alpha=0)$, le matériau est isotrope. Les positions initiales et les tailles des surfaces de charge reflètent l'histoire des sollicitations antérieures du matériau. L'équation d'une surface de charge est donnée par:

$f(\underline{\alpha})=\frac{3}{2}(s-\bar{p} \alpha):(\underline{s}-\bar{p} \underset{\alpha}{\alpha})-m^{2} \bar{p}^{2}$

s. représente le tenseur des contraintes déviatoriques,

$$
\overline{\mathrm{p}}=\mathrm{p}+\mathrm{C}^{\prime} \operatorname{cotg} \phi
$$

$\mathrm{m} \overline{\mathrm{p}}$ représente la taille de la surface de charge dans le plan déviatorique. 


\subsection{Loi d'écoulement plastique}

A chaque surface de charge, est associée une loi d'écoulement plastique. Cependant, la déformation déviatorique est associée, autrement dit le vecteur vitesse de déformation déviatorique est normal à la surface de charge ; la déformation volumique est non associée. Cette non-associativité permet de refléter le comportement initialement contractant puis ultérieurement dilatant des sols lors des essais de cisaillement. La loi de comportement intègre ainsi la notion de seuil caractéristique défini par LUONG [6].

\subsection{Loi d'écrouissage}

L'écrouissage adopté est de type cinématique et pure. ment déviatorique.

Pour permettre l'ajustement de la loi d'écrouissage à n'importe quel type de données expérimentales, on utilise le concept de surfaces de charge multiples incluses les unes dans les autres. Lors de l'écoulement plastique, le point $M$ représentatif de l'état de contrainte entraine successivement les différentes surfaces de charge qu'il rencontre. Lorsqu'il atteint la surface $f_{i}$, toutes les surfaces intérieures $\left(f_{1-1}, \ldots, f_{1}\right)$ ont été translatées et sont tangentes entre elles et à $f_{i}$ au point $M$ (fig. 3 ). Lorsque le point $M$ est situé sur la surface $f_{1}$, toutes les surfaces intérieures sont inactives et seuls les paramètres de déformation plastique de la surface $f_{i}$ sont pris en compte. Si le point $M$ quitte la surface $f_{1}$ et se déplace à l'intérieur de celleci, le comportement du sol sera de nouveau élastique jusqu'à ce qu'il rencontre de nouveau la surface $f_{1}$.

\subsection{Détermination des paramètres de la loi de comportement}

La loi de comportement décrite précédemment comporte :

- trois paramètres de définition du comportement élastique :

- $G$ module de cisaillement

- B module de déformation volumique

- $\mathrm{n}$ exposant décrivant la dépendance des modules élastiques et plastiques sur l'état de contrainte (équation 2.1) ;

- six paramètres plastiques par surface de charge: $\alpha$ paramètre de position de la surface de charge $\mathrm{m}$ paramètre de taille de la surface de charge $\mathrm{H}_{\mathrm{c}}$ module plastique en compression $\mathrm{H}_{e}$ module plastique en extension $\bar{\eta}_{e}, \bar{\eta}_{e}$ paramètres de définition du seuil caractéristique en compression et en extension.

En toute théorie, la détermination de l'ensemble des paramètres définissant le modèle de comportement peut s'effectuer à partir de deux essais triaxiaux drainés, l'un correspondant à un chargement en compression, l'autre à un chargement en extension. Le nombre de surfaces de charge retenu est fonction du degré d'approximation linéaire des courbes effortdéformation. Plus ce nombre est élevé, meilleure est Papproximation ; dans l'étude présente, on a retenu cinq surfaces de charge.
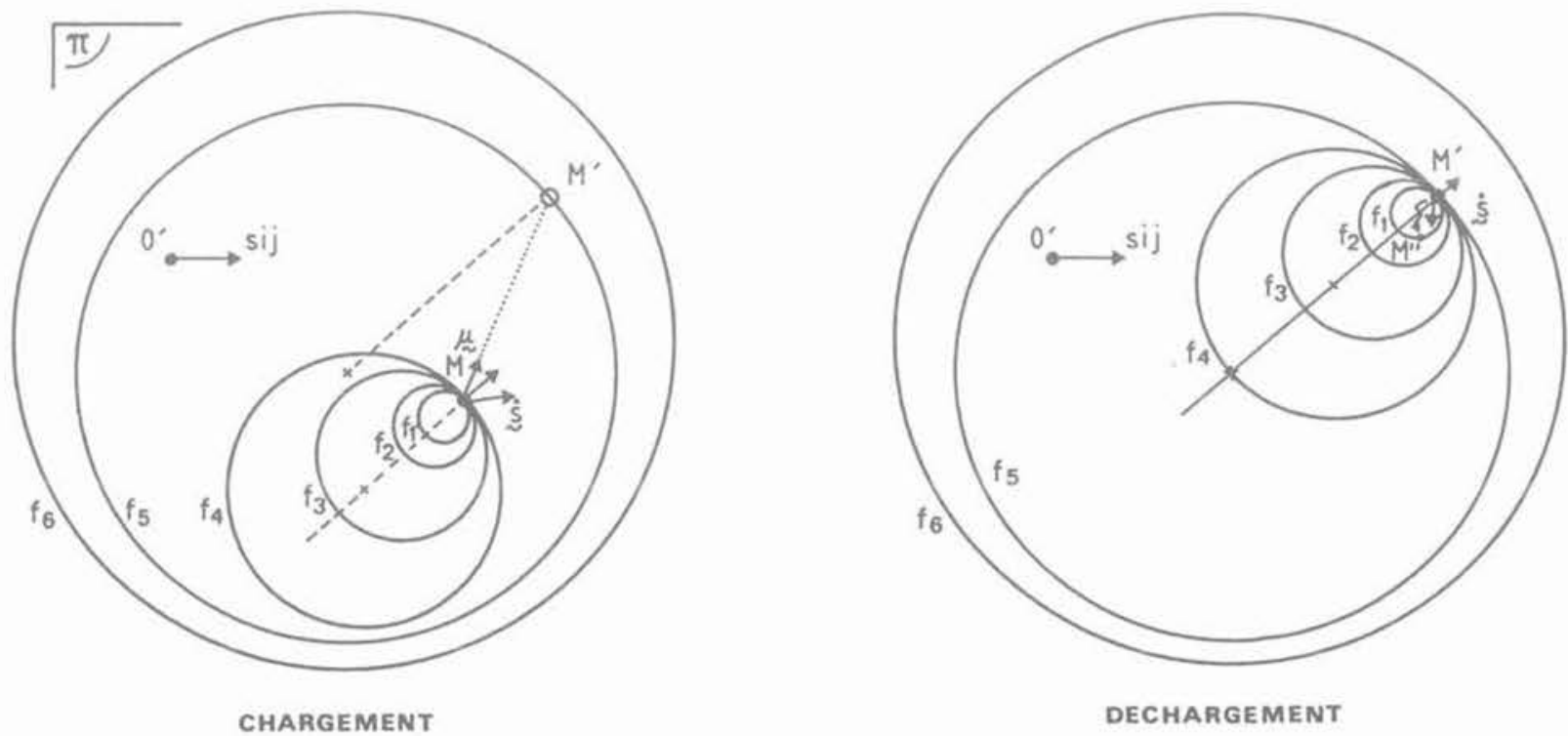

Fig. 3. - Ecrouissage cinématique du modèle à surfaces multiples.

Fig. 3. - Kinematic hardening of the multiple yield surface model 


\section{PARAMÈTRES DE LA LOI}

\section{DE COMPORTEMENT POUR L'ARGILE} DU GAULT INFÉRIEUR

L'argile retenue pour la simulation (Gault inférieur de Penly) est fortement surconsolidée $(\mathrm{OCR}=7$ ) et présente in situ un état de contraintes effectives homogène sur une forte épaisseur, du fait du gradient hydraulique ascendant créé par la présence de la nappe artésienne. In situ, les contraintes sont estimées à :

- contrainte verticale effective $\quad \sigma_{2}^{\prime}=0.7 \mathrm{MPa}$

- contrainte horizontale effective $\sigma_{x}^{\prime}=\sigma_{y}^{\prime}=0,95 \mathrm{MPa}$.

Les essais triaxiaux de laboratoire ayant servi à la détermination des paramètres du modèle ont été réalisés sur des éprouvettes consolidées anisotropiquement sous l'état de contraintes défini précédemment. Usuellement, la détermination des paramètres du modèle s'effectue à partir d'essais triaxiaux consolidés drainés. Cependant, compte tenu de la faible perméabilité des éprouvettes $\left(\mathrm{K}=10^{-12} \mathrm{~m} / \mathrm{s}\right)$, il est probable que les essais drainés, bien qu'effectués à une vitesse d'écrasement de $1 \mu \mathrm{m} / \mathrm{mn}$, ne permettaient pas une dissipation totale de la pression interstitielle. faussant ainsi les variations de volume mesurées. Il a donc été décidé d'ajuster les paramètres de la loi de comportement à partir d'essais triaxiaux consolidés non drainés. Les paramètres obtenus sont les suivants :

- module de cisaillement $\mathrm{G}=73 \mathrm{MPa}$ pour une pression de référence $p_{1}=0,87 \mathrm{MPa}$, correspon-

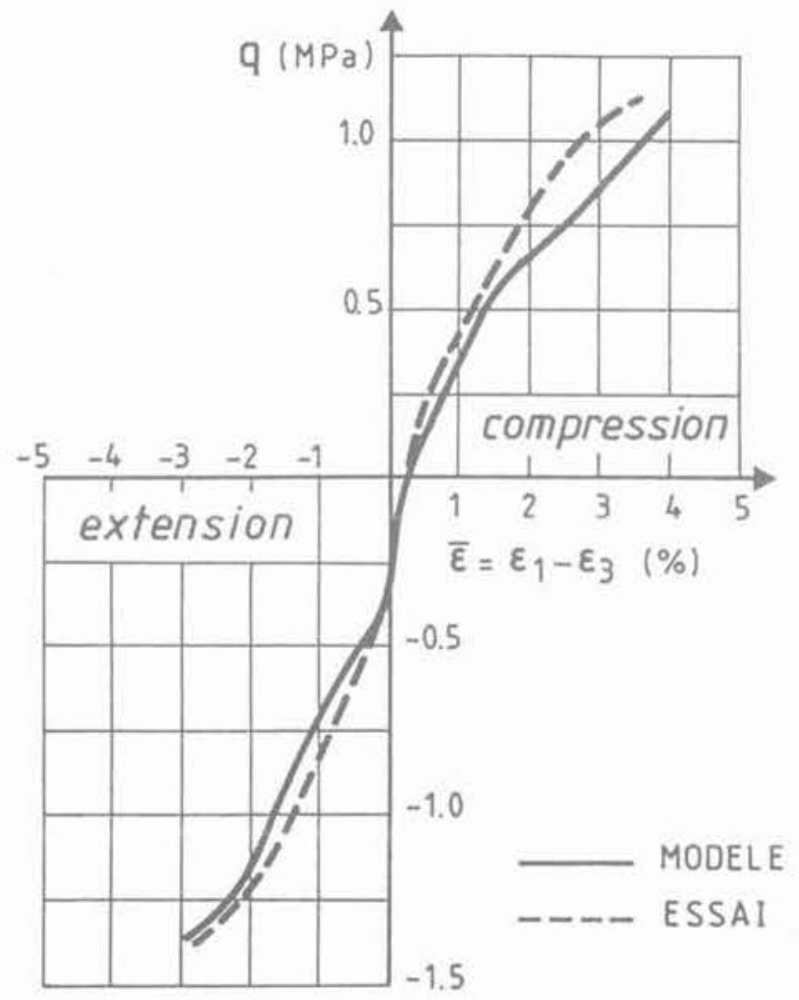

Fig. 4a. - Gault inférieur - Essais triaxiaux non drainés Calage du modàle.

Fig. $4 a$ - Lower Gault - undrained triaxial tests. Determination of the model parameters. dant à la contrainte moyenne effective de consolidation :

- module de compressibilité volumétrique $\mathrm{B}=30$ $\mathrm{MPa}$ pour une contrainte effective moyenne de consolidation $\mathrm{p}_{1}=0,87 \mathrm{MPa}$;

- exposant $\mathrm{n}=0,53$ déterminé à partir d'essais à la colonne résonante;

- les paramètres plastiques associés à chaque surface de charges sont donnés dans le tableau 1.

Les courbes effort-déformation obtenues sur l'argile du Gault inférieur présentant une grande portion linéaire. seules cinq surfaces de charges ont été retenues. Par ailleurs, il n'a pas été jugé nécessaire de retenir des valeurs du coefficient $\frac{j}{\eta}$ différentes pour chaque surface de charge. Une valeur unique a été retenue pour l'ensemble des cinq surfaces de charge:

$$
\bar{\eta}_{e}=-0,1 ; \bar{\eta}_{c}=0,75 \text {. }
$$

Tableau 1. Paramètres des surfaces de charges Table 1. Load surfaces parameters

\begin{tabular}{|c|c|c|r|r|}
\hline surface & $\alpha$ & $n$ & $\frac{H_{c}^{\prime}}{p_{1}}$ & $\frac{H^{\prime}}{p_{1}}$ \\
\hline 1 & $-0,165$ & 0,037 & 1781 & 2501 \\
2 & $-0,190$ & 0,127 & 92 & 433 \\
3 & $-0,076$ & 0,407 & 30 & 30 \\
4 & $-0,296$ & 0,704 & 26 & 37 \\
5 & $-0,125$ & 0,855 & 0 & 0 \\
\hline
\end{tabular}

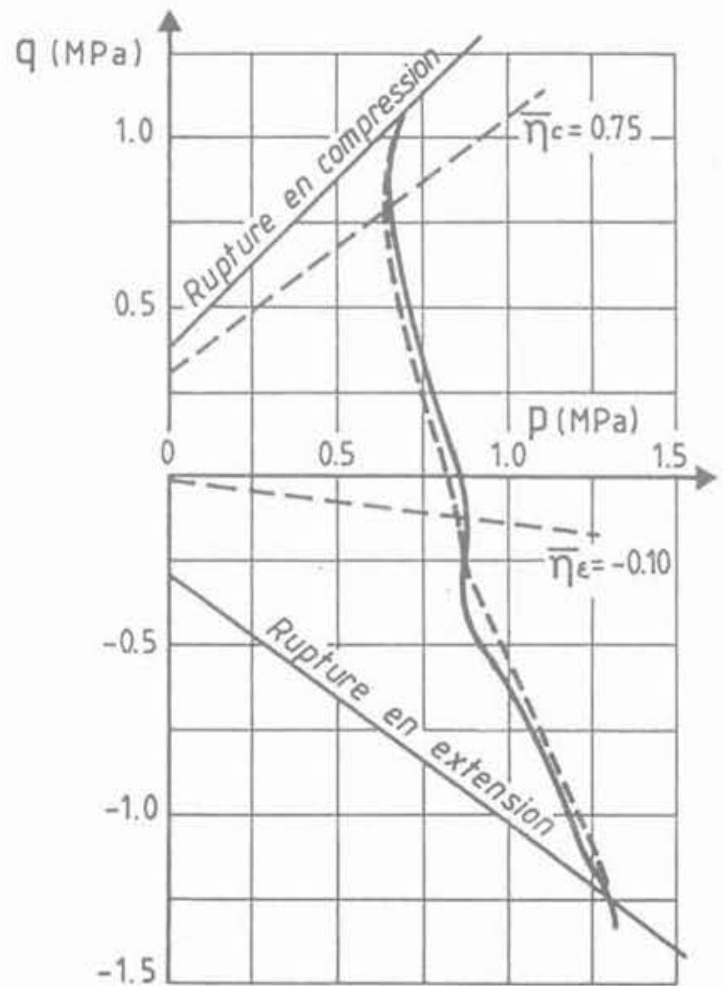

Fig. 4b. - Gault inférieur - Essais triaxiaux non drainés Calage du modèle.

Fig. $4 b$ - Lower Gault - undrained triaxial tests - Comparison between model prediction and tests results. 
La dernière surface de charge $\left(n^{\circ} 5\right)$ joue le rôle d'une surface de rupture. En conséquence, ses modules de déformation plastique en compression et en extension sont nuls.

Les figures $4 \mathrm{a}$ et $4 \mathrm{~b}$ présentent les résultats du calage du modèle de comportement en terme de courbe effort-déformation (figure 4a) et chemin de contrainte effective dans le plan $p$ (contrainte moyenne) $q$ (déviateur) (figure 4b)

La simulation de l'essai triaxial consolidé drainé correspondant à la même éprouvette est comparée sur la figure 5 aux résultats expérimentaux obtenus. La figure $5 \mathrm{a}$ montre que la courbe effort-déformation est correctement approchée, par contre, les variations de volume estimées par le modèle sont beaucoup plus importantes que celles obtenues lors de l'essai (fig. $5 \mathrm{~b}$ ). Ce point a été discuté précédemment.

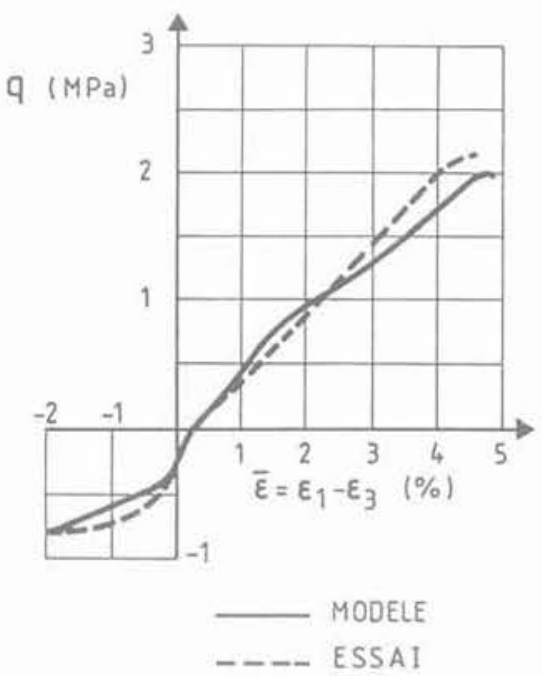

Fig. 5a - Gault inférieur - Essais triaxiaux.

Fig. 5a. - Lower Gault - drained triaxial tests - Comparison between model prediction and tests results

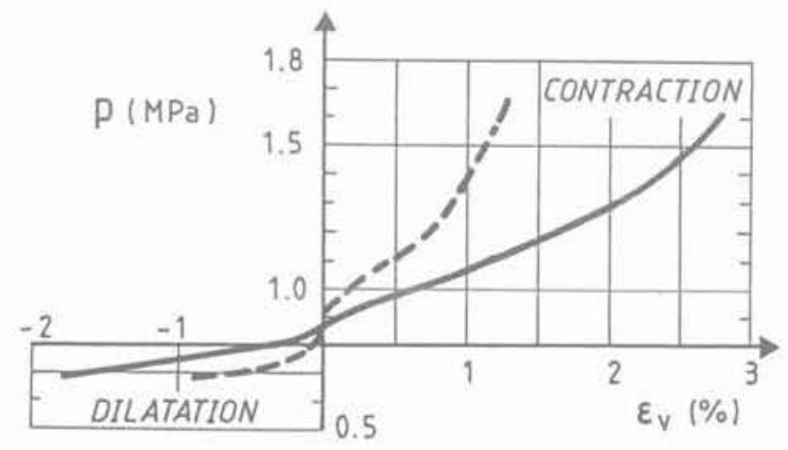

Fig. 5b. - Gault inférieur - Essais triaxiaux drainés.

Fig. 5b. - Lower Gault - drained triaxial tests - Comparison between model prediction and tests results.

\section{VALIDATION DU MODÈLE DE COMPORTEMENT}

Avec le jeu de paramètres défini au paragraphe précédent, il est possible de simuler d'autres types d'essais de laboratoire ou in situ correspondant à des états et des trajets de contrainte différents de ceux de l'essai ayant servi à la détermination des paramètres du modèle.

\subsection{Essais triaxiaux}

Deux autres séries d'essais triaxiaux consolidés non drainés, réalisées sous des états de contraintes diffé. rents $\left(\sigma_{1}=0,37 \mathrm{MPa}, \sigma_{3}=0,50 \mathrm{MPa}\right.$ et $\sigma_{1}=1,1$ $\left.\mathrm{MPa}, \sigma_{3}=1.5 \mathrm{MPa}\right)$, ont été simulées numériquement. La comparaison avec les données expérimentales est présentée sur la figure 6 . On constate une très bonne concordance entre simulation et expérience, y compris pour les trajets de contrainte effective dans le plan $\mathrm{p}-\mathrm{q}$.

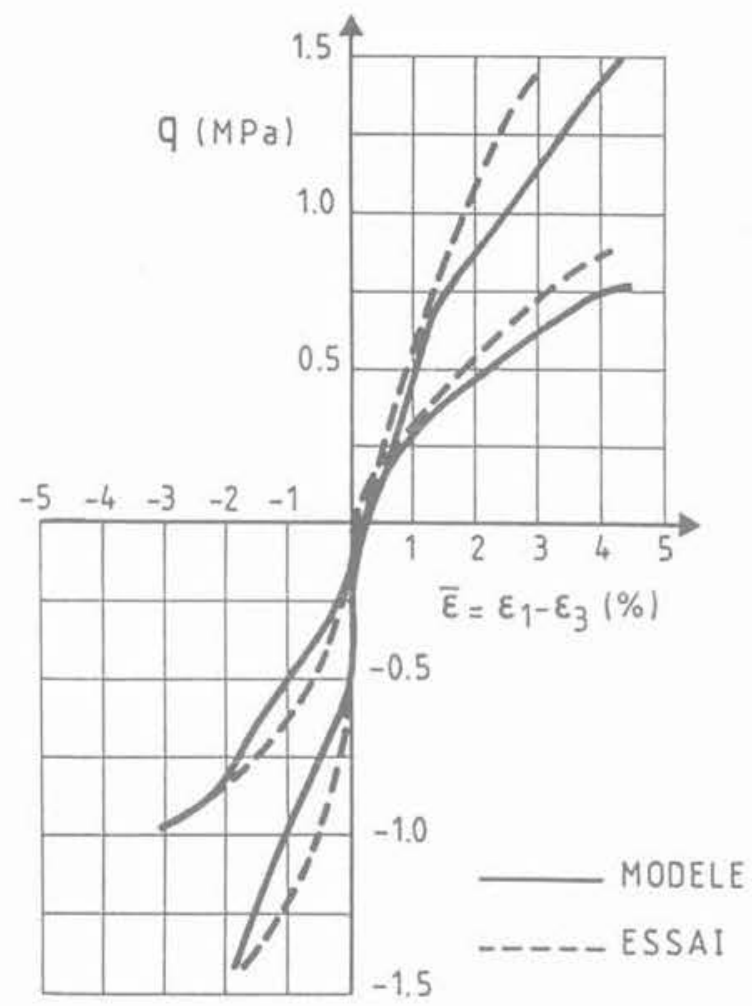

Fig. 6a. - Gault inférieur - Essais triaxiaux non drainés Fig. 6a. - Lower Gault - Undrained triaxial tests - Comparison between model prediction and tests results. 


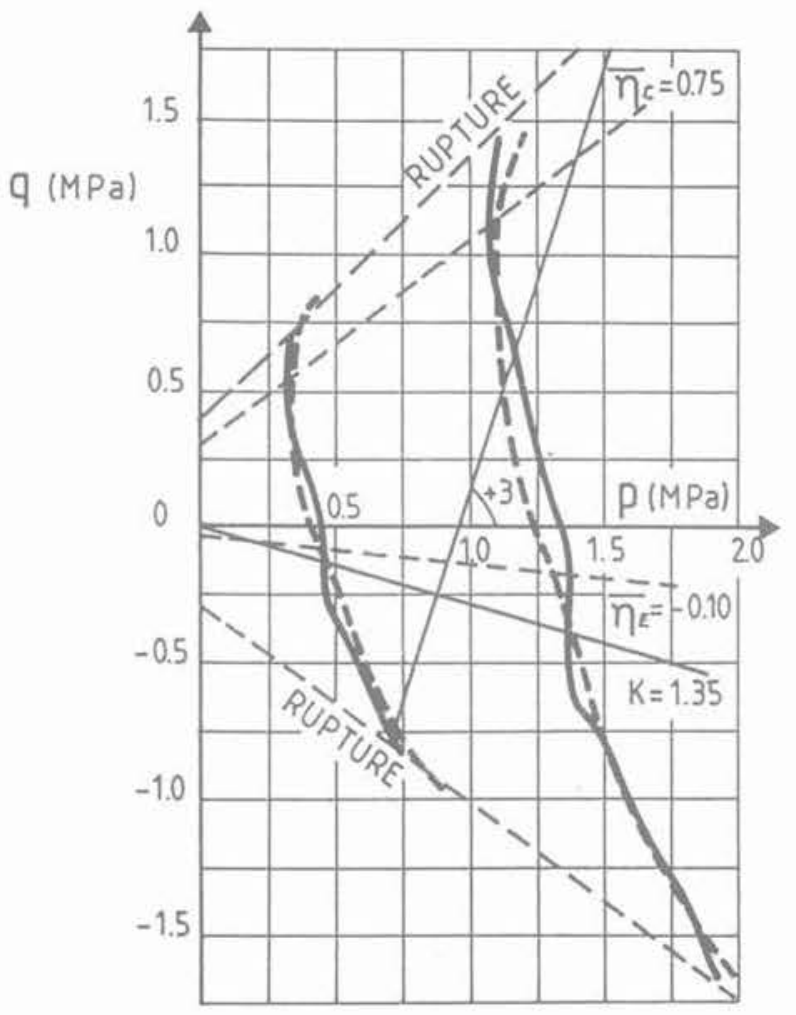

Fig. 6b. - Gault inférieur. Essais triaxiaux non drainés. Fig. 6b. - Lower Gault - Undrained triaxial tests.

\subsection{Essais œdométriques}

La simulation de la mise en place dans l'œdomètre a été faite en supposant que l'échantillon s'est détendu, lors du prélèvement, en condition non drainée; l'eau interstitielle est supposée parfaitement désaérée et présente un module de compressibilité volumique de $2000 \mathrm{MPa}$. Le prélèvement de l'échantillon a pour effet d'annuler les contraintes totales appliquées in situ créant de ce fait, une pression interstitielle négative $\mathrm{u}=-0,82 \mathrm{MPa}$. Le matériau se trouve en fin de prélèvement dans un état de contrainte effective isotrope ( $\left.q=0, p^{\prime}=0,82 \mathrm{MPa}\right)$. A partir de cet état, l'essai œedométrique proprement dit est simulé en appliquant à l'échantillon, en condition drainée, un accroissement de contrainte verticale $\sigma_{1}$, en maintenant la déformation radiale nulle.

La comparaison entre la courbe calculée et la courbe cedométrique mesurée est donnée sur la figure 7. Elle fait apparaître un décalage entre les deux courbes. $\mathrm{Ce}$ décalage peut s'expliquer par la formation de bulles d'air causée par la détente de l'échantillon en cours de prélèvement ; cet air est dissous dans l'eau interstitielle ( $2 \%$ en volume suivant la loi de Henry), le nombre de moles d'air dissoutes étant proportionnel à la pression absolue du fluide interstitiel. Lors de la détente, le fluide interstitiel devient biphasique (liquide + gaz) et se comporte comme un fluide à module variable. La pression interstitielle négative de leau sera d'autant moins importante en valeur absolue que le laps de temps écoulé entre le prélèvement et l'application de la charge œedométrique aura été long. L'état de contrainte initial étant isotrope, on peut supposer

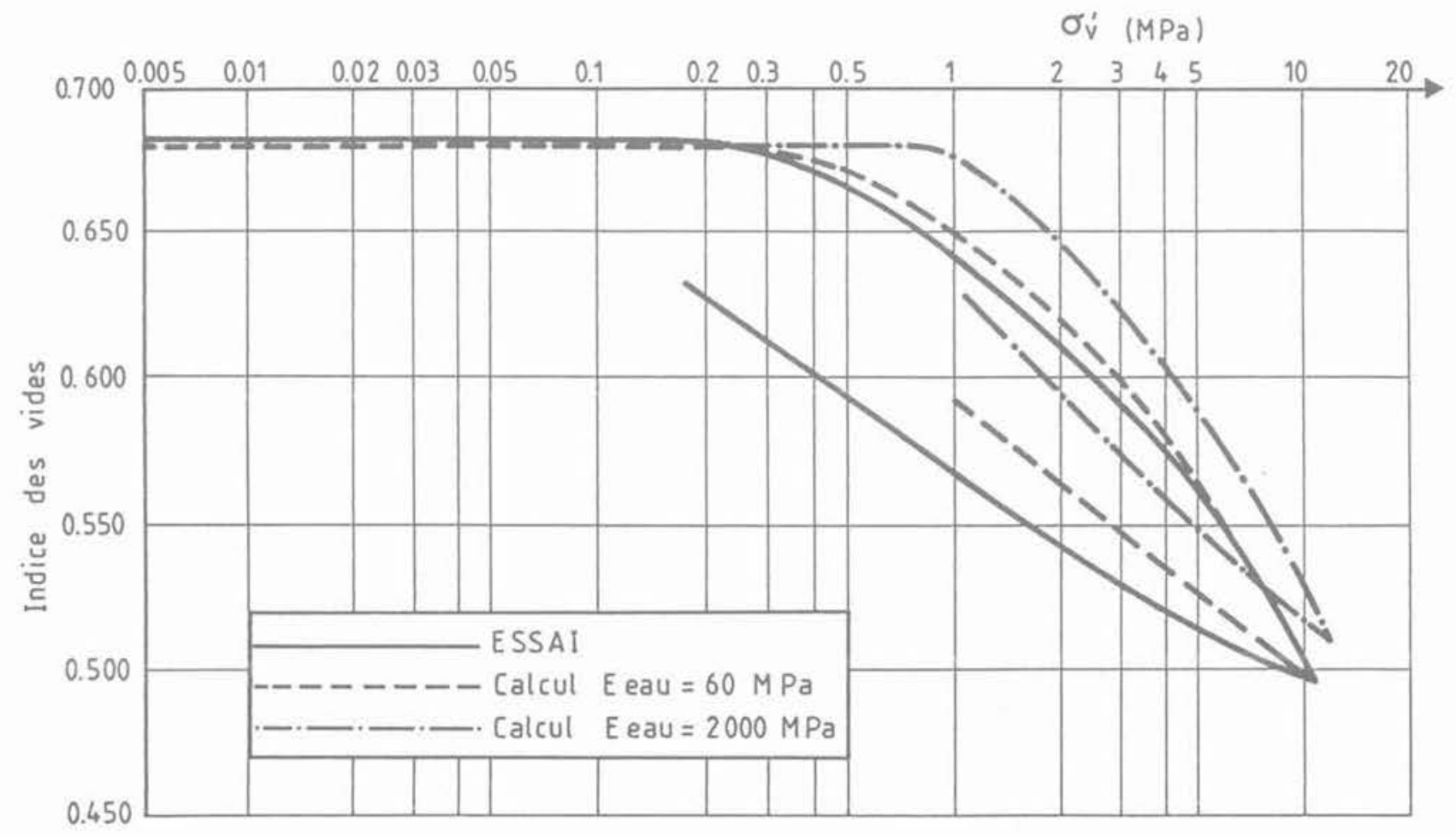

Fig. 7. - Gault inférieur. Essai œdométrique.

Fig. 7. - Lower Gault - Compressibility tests - Comparison

between model prediction and tests results. 
que la contrainte effective moyenne est de $0,3 \mathrm{MPa}$ au vu de la courbe expérimentale. Cet état de contrainte est obtenu lors de la simulation du prélèvement en attribuant au fluide interstitiel un module de 60 $\mathrm{MPa}$. La dilatation théorique de l'échantillon due à l'apparition d'air libre est alors de $10^{-2}$. L'essai cedométrique calculé à partir de cet état de contrainte (u $=-0,3 \mathrm{MPa}$ ) est représenté sur la figure 7. La concordance avec la courbe expérimentale est très bonne, aussi bien en charge qu'en décharge.

\subsection{Essais pressiométriques}

Sur le site de Penly, 18 essais pressiométriques standards et cycliques ont été réalisés. On se réfèrera dans la suite à l'essai réalisé à la cote - 74 NGF correspondant à la cote de prélèvement des échantillons ayant servi au calage du modèle de comportement. Pour tous les essais pressiométriques réalisés à Penly, la procédure de réalisation de l'essai diffère de la procédure habituelle et est rappelée ci-dessous.

Le forage, exécuté au taillant avec injection, à un diamètre de $66 \mathrm{~mm}$ dans l'argile du Gault. La boue de forage est lestée à la baryte et a pour densité 1,7, ceci afin de limiter les déformations radiales du trou et d'équilibrer la pression de la nappe artésienne des sables verts de l'Albien. Pour réduire l'intervalle de temps séparant la perforation de l'exécution des essais pressiométriques, le forage est effectué par passes de $2 \mathrm{~m}$. Chaque passe comporte la réalisation d'un essai standard (chargement monotone) et d'un essai cyclique. Pour l'essai standard, le milieu de la sonde est positionné à $0,50 \mathrm{~m}$ du fond de trou. Au-dessus de la sonde, le train de tiges creuses $\varnothing 63 \mathrm{~mm}$ limite la convergence du diamètre du forage de 66 à 63 $\mathrm{mm}$. La sonde pressiométrique de type AX a un diamètre $\varnothing=44 \mathrm{~mm}$ et une longueur de $0,60 \mathrm{~m}$; elle est protégée par un tube fendu de diamètre $\varnothing$ $=63 \mathrm{~mm}$. La cellule de mesure a une hauteur de $0,36 \mathrm{~m}$ et un volume au repos de $535 \mathrm{~cm}^{3}$. Les cellules de garde ont une hauteur de $0,12 \mathrm{~m}$. L'essai pressiométrique est conduit par incrément de pression de $0,5 \mathrm{MPa}$, chaque palier étant maintenu une minute, exception faite pour le premier palier maintenu $5 \mathrm{~mm}$. La courbe expérimentale $v=f(p)$ a été tracée en tenant compte des corrections dues à l'inertie de la sonde, à la dilatation des circuits hydrauliques, et à la pression de la colonne d'eau alimentant la sonde. Elle est donnée sur la figure 8.

L'essai pressiométrique a été simulé par un calcul en éléments finis, prenant en compte la présence de l'eau interstitielle (milieu poreux biphasique). La perméabilité in situ du Gault inférieur est estimée à $10^{-9} \mathrm{~m} / \mathrm{s}$ et le module de l'eau est pris égal à $2000 \mathrm{MPa}$. Le choix de la valeur de la perméabilité, différente de celle mesurée sur éprouvette $\left(10^{-12} \mathrm{~m} / \mathrm{s}\right)$, résulte de l'interprétation des déformations de la galerie de rejet de la centrale de Penly. Cette valeur rend compte de la perméabilité en grand de l'argile du Gault inférieur. La porosité de la couche d'argile est prise égale à 0,37 et la densité des grains est de 2,71.

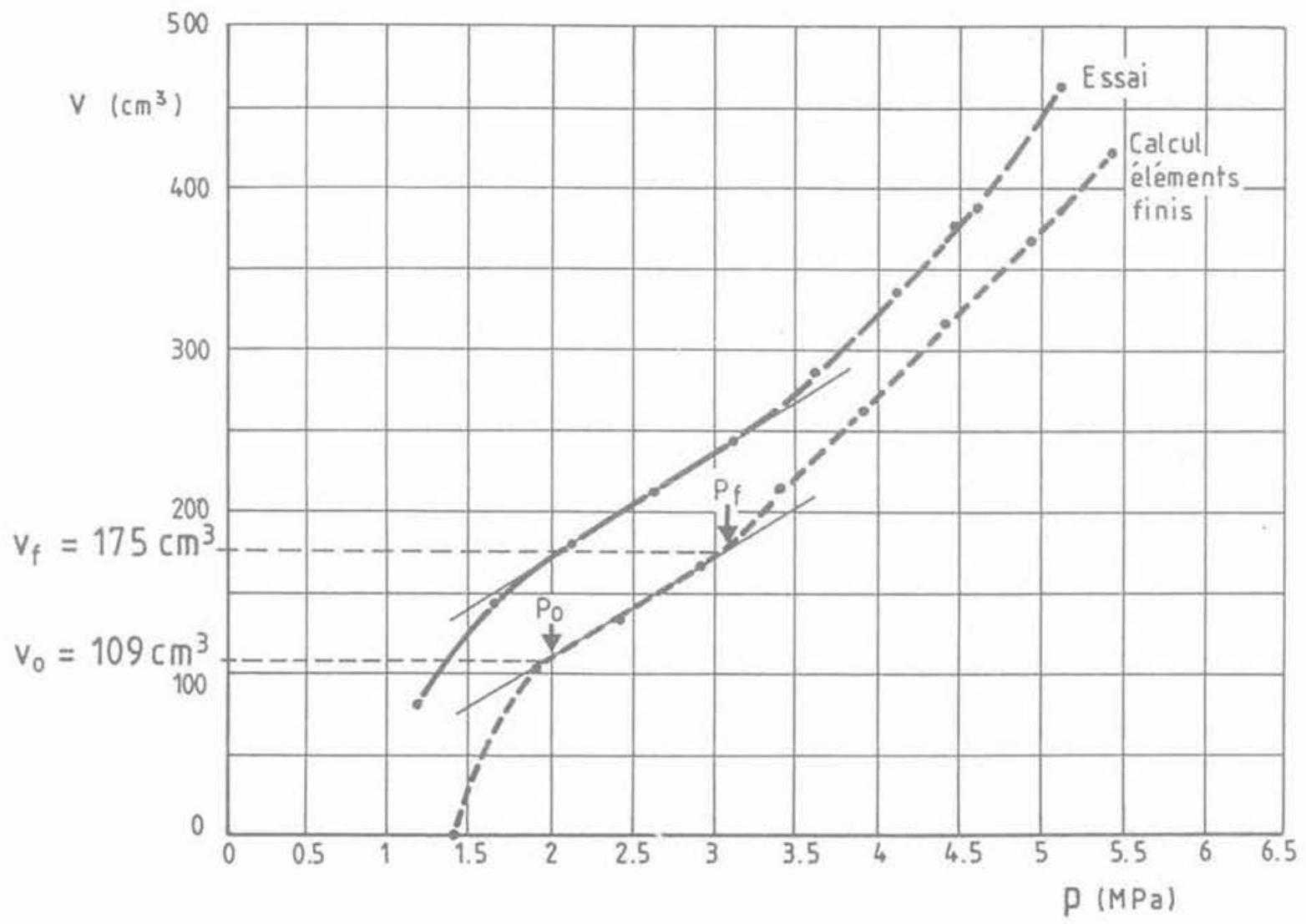

Fig. 8. - Essai pressiométrique réalisé à Penly. Cote : - 74 NGF.

Fig. 8. - Pressuremeter test at Penly - elevation : - 74 NGF. 
Les étapes de calcul numérique sont les suivantes: - initialisation des contraintes effectives $\sigma^{\prime}{ }_{v}=0,6 \mathrm{MPa}$,

$\sigma_{\mathrm{h}}^{\prime}=0,8 \mathrm{MPa}$;

- simulation de la passe de forage par diminution des contraintes appliquées sur la paroi depuis la valeur initiale jusqu'à une valeur représentant la pression créée par la colonne de boue (1,33 $\mathrm{MPa})$. Cette étape est effectuée en $15 \mathrm{mn}$;

- maintien du forage dans son état actuel pendant $60 \mathrm{mn}$ (temps nécessaire pour remonter le taillant et installer la sonde pressiométrique) ;

- application des pressions exercées par la sonde dans la cellule de mesure et les cellules de garde (incrément de $0,5 \mathrm{MPa}$ par palier de $1 \mathrm{mn}$, sauf le premier palier maintenu pendant $5 \mathrm{mn}$ ).

La courbe $(p, v)$ obtenue par le calcul est représentée sur la figure 8. De cette courbe, on déduit les paramètres suivants :

- état initial $\mathrm{p}_{0}=1,98 \mathrm{MPa}, \mathrm{v}_{0}=109 \mathrm{~cm}^{3}$

- fin de la phase pseudo-élastique $\mathrm{p}_{\mathrm{f}}=3,08 \mathrm{MPa}$,

$v_{f},=175 \mathrm{~cm}^{3}$

- module pressiométrique $\mathrm{E}_{\mathrm{m}}=30 \mathrm{MPa}$

La pression limite $\mathrm{p}_{1}$ a été estimée par différentes méthodes (Van Wambeke et Henricourt, méthode LCPC), elle varie entre 7,3 $\mathrm{MPa}$ et $10,6 \mathrm{MPa}$.

La comparaison entre la courbe calculée et la courbe expérimentale montre la similitude existant entre les deux. Elles se déduisent l'une de l'autre par une translation de volume d'environ $50 \mathrm{~cm}^{3}$. Cette valeur est de l'ordre de grandeur de l'erreur commise en évaluant le contact tube-sonde. En se référant aux cotes fournies, la sonde au repos a un diamètre de $44 \mathrm{~mm}$ et le tube fendu un diamètre de $49 \mathrm{~mm}$. La mise au contact sonde-tube nécessiterait une dilatation de la sonde de $132 \mathrm{~cm}^{3}$ alors que les étalonnages ont conduit à une dilatation de $115 \mathrm{~cm}^{3}$. Ce décalage de courbe, quelle qu'en soit l'origine n'a aucune influence sur l'interprétation de l'essai. Le tableau 2 présente la comparaison, pour chacun des paramètres déduits de l'essai pressiométrique, des valeurs calculées à l'aide du modèle numérique et des valeurs effectivement mesurées. La dernière colonne donne pour l'ensemble des essais réalisés dans l'argile du Gault inférieur à Penly, la moyenne des valeurs mesurées.

Tableau 2. Paramètres des essais pressiométriques Table 2. Pressuremeter tests parameters

\begin{tabular}{|l|c|c|c|}
\hline & $\begin{array}{c}\text { Valeur calculée } \\
\text { à la cote } \\
-74 \text { NGF }\end{array}$ & $\begin{array}{c}\text { Essais à } \\
\text { la cote } \\
-74 \text { NGF }\end{array}$ & $\begin{array}{c}\text { Moyenne } \\
\text { déduite } \\
\text { des mesures }\end{array}$ \\
\hline$E_{m}(\mathrm{MPa})$ & 30 & 31 & 33 \\
$\mathrm{p}_{0}(\mathrm{MPa})$ & 2,0 & 2,0 & 1,6 \\
$\mathrm{p}_{f}(\mathrm{MPa})$ & 3,0 & 3,2 & 2,9 \\
$\mathrm{p}_{1}^{*}(\mathrm{MPa})$ & 10,6 & 8,9 & 7,7 \\
$\mathrm{p}_{1}^{*}$ * $(\mathrm{MPa})$ & 7,3 & 6,5 & 6,2 \\
\hline
\end{tabular}

- Méthode LCPC

* Méthode Van Wambeke et Henricourt

\section{5. ÉVALUATION DU TASSEMENT D'UN RÉACTEUR NUCLÉAIRE FONDÉ SUR UNE COUCHE HOMOGÈNE D'ARGILE DU GAULT INFÉRIEUR}

La fondation circulaire choisie est une plaque rigide de rayon $25 \mathrm{~m}$ surmontant une couche d'argile homogène d'épaisseur $50 \mathrm{~m}$. Le tassement de cette fondation a été évaluée :

- à partir de l'interprétation classique d'essais œedométriques et pressiométriques simulés numériquement :

- par un calcul numérique en éléments finis.

\subsection{Evaluation à partir des essais}

\subsubsection{Essais œdométriques}

L'évaluation des tassements par la méthode œdométrique suppose que l'on dispose d'un certain nombre d'essais effectués sur des échantillons prélevés à différentes profondeurs sous la fondation. La simulation numérique de ces essais a été effectuée de façon identique à celle décrite au paragraphe 4. L'eau interstitielle a été supposée désaérée avec un module $\mathrm{B}=$ $2000 \mathrm{MPa}$. Les essais simulés aux profondeurs de $5 \mathrm{~m}, 10 \mathrm{~m}, 20 \mathrm{~m}$ et $30 \mathrm{~m}$ sous la fondation sont donnés sur la figure 9.

Le tassement a été évalué par la méthode cedométrique en discrétisant la couche de fondation en un certain nombre de sous-couches et en calculant la

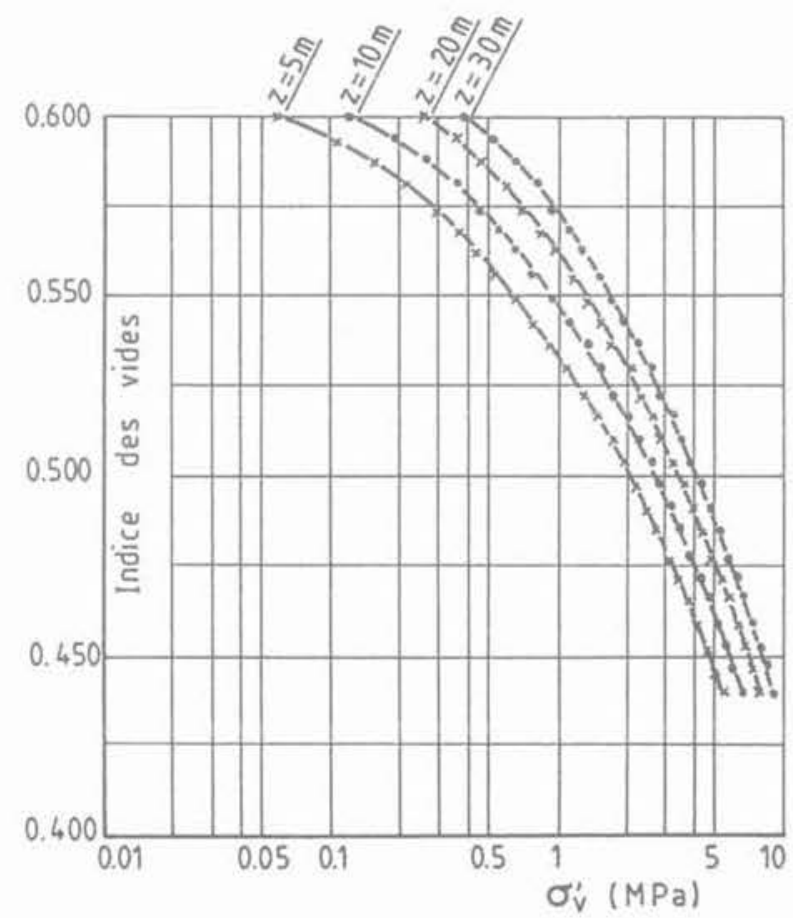

Fig. 9. - Edomètres sous la fondation - Calcul numérique. Fig. 9. - Compressibility tests under the foundation numerical calculation 
répartition des contraintes verticales dans l'axe de la fondation par les formules de Boussinesq. La courbe de tassement en fonction de la charge appliquée à la fondation, est donnée sur la figure 13.

\subsubsection{Essais pressiométriques}

Trois essais pressiométriques ont été simulés aux profondeurs de $10 \mathrm{~m}, 20 \mathrm{~m}$ et $30 \mathrm{~m}$ sous la fondation. Le résultat de ces simulations est donné sur la figure 10. La simulation a été faite en supposant une procédure standard de réalisation de l'essai pressiométrique. Le forage de diamètre $66 \mathrm{~mm}$ est réalisé en une seule passe avec une boue de forage de densité 1,2 . Les essais pressiométriques sont réalisés 24 heures après la fin de foration. Les paliers de charge sont de $1 \mathrm{mn}$ et la pression dans les cellules de garde est inférieure de $0.1 \mathrm{MPa}$ à celle dans la cellule de mesure.

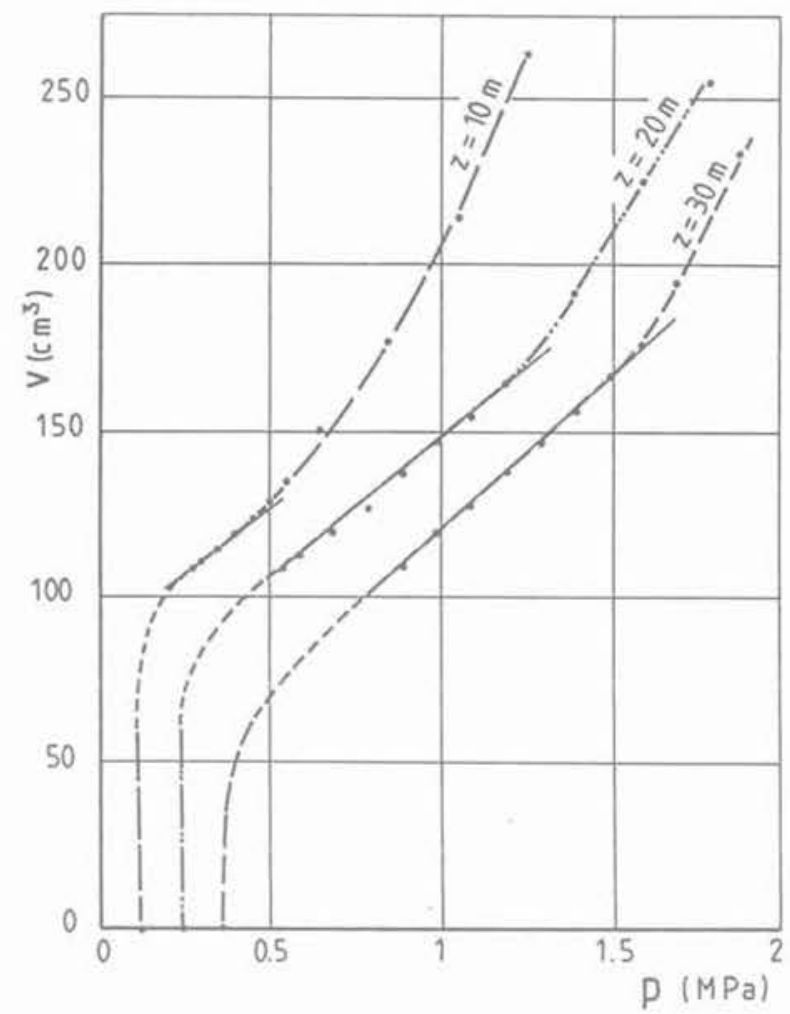

Fig. 10. - Pressiomètres sous la fondation-Calcul numérique. Fig. 10. - Pressuremeter tests under the foundation numerical calculation

Le module pressiométrique déduit des essais simulés numériquement varie de $19 \mathrm{MPa}$ à $30 \mathrm{~m}$ de profondeur à $21 \mathrm{MPa}$ à $10 \mathrm{~m}$ de profondeur.

Un essai pressiométrique cyclique a également été simulé à la profondeur de $20 \mathrm{~m}$. Son résultat est donné sur la figure 11. En première charge, le module pressiométrique vaut $20,8 \mathrm{MPa}$, après le premier chargement, les modules en charge et en décharge sont assez voisins, compris entre 45 et 50 $\mathrm{MPa}$. Le rapport entre le module de première charge et le module de première décharge est de 0,46 . Ce rapport est très proche de la valeur du coefficient rhéologique $\alpha$ déterminé à partir du rapport $E /\left(p_{1}\right.$

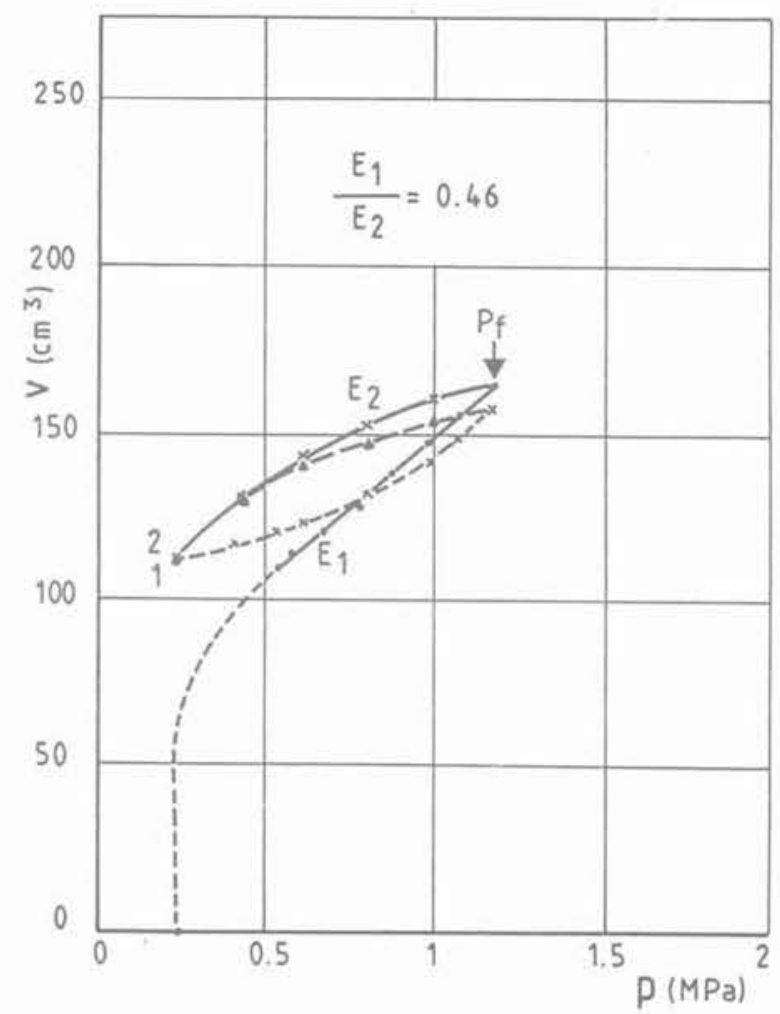

Fig. 11. - Pressiomètre cyclique sous la fondation. $z=20 \mathrm{~cm}$

Fig. 11. - Cyclic pressuremeter tests under the foundation. $z=20 \mathrm{~m}$

- $p_{0}$ ) qui est égal à 0,5 . Ceci est conforme aux observations expérimentales, BAGUELIN et al [1].

Le tassement déduit des essais pressiométriques a été évalué en suivant les règles pressiométriques usuelles. Dans l'application de la formule pressiométrique, le paramètre essentiel est le coefficient rhéologique $\alpha$. Les tassements évalués pour des valeurs $\alpha=0,5$ et $\alpha=1,0$ sont données, en fonction de la charge appliquée, sur la figure 13. La valeur $\alpha=0,5$ est celle que l'on déduirait du rapport $E /\left(p_{1}-p_{0}\right)=$ 7 ou du rapport $E_{1}$ (module de première charge) / $\mathrm{E}_{2}$ (module de première recharge) $=0,46 . \mathrm{La}$ valeur $\alpha=1$ est celle que l'on retiendrait normalement en ne considérant que la nature du matériau : argile surconsolidée. Elle conduit à un tassement un peu plus élevé que celui de la méthode cedométrique pour les fortes valeurs de la surcharge.

On doit noter une contradiction entre le caractère surconsolidé de l'argile et la valeur très faible du rapport $E /\left(p_{1}-p_{0}\right)$, qui traduirait a priori un remaniement du sol lors de l'essai. Mais la simulation numérique donne la même valeur du rapport $E /\left(p_{1}-p_{0}\right)$, ce qui semblerait indiquer qu'il s'agit là d'un résultat propre à l'argile du Gault.

\subsection{Evaluation numérique du tassement}

Le maillage en éléments finis, axisymétrique, ayant servi à cette évaluation, est présenté sur la figure 12. Les nceuds de la frontière inférieure du modèle sont 


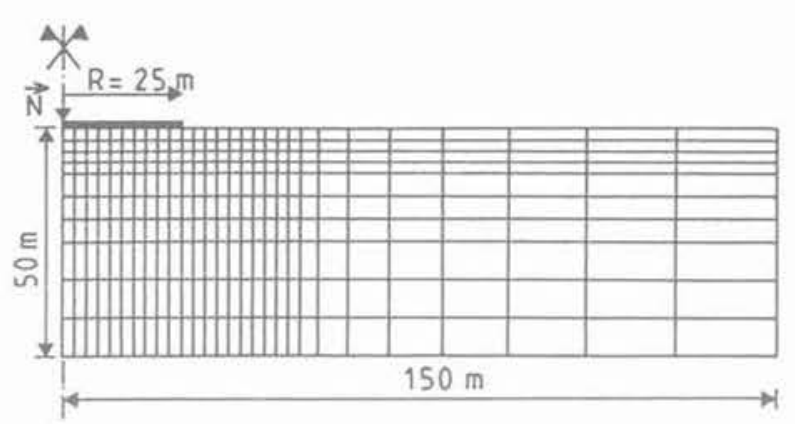

Fig. 12. - Maillage éléments finis.

Fig. 12. - Finite element mesh.

bloqués. Sur les frontières latérales du modèle (axe de symétrie et bord extérieur), seuls les déplacements verticaux sont autorisés.

Ce maillage a été validé en effectuant des calculs élastiques pour une fondation circulaire chargée axialement et en comparant les résultats obtenus aux solutions théoriques publiées dans la littérature. Les erreurs relatives étaient inférieures à $5 \%$ pour le tassement, quelle que soit la valeur du coefficient de Poisson comprise entre 0 et 0,5 , et inférieure à $10 \%$ pour la distribution des contraintes verticales sous la fondation.

Le calcul du tassement de la fondation a été conduit de façon incrémentale, pour prendre correctement en compte les non-linéarités de comportement du sol. La première étape a consisté en une initialisation des contraintes effectives initiales $\sigma_{v}^{\prime}$ et $\sigma_{\mathrm{h}}^{\prime}$. La contrainte effective initiale $\sigma_{v}^{\prime}$ est égale au poids déjaugé des terrains et la contrainte horizontale $\sigma_{\mathrm{h}}^{\prime}$ a été prise égale à 1.35 fois la contrainte verticale effective sur toute l'épaisseur de la couche d'argile surconsolidée. Les paramètres de la loi de comportement sont ceux qui ont été définis au paragraphe 3 . La courbe de tassement est donnée sur la figure 13.

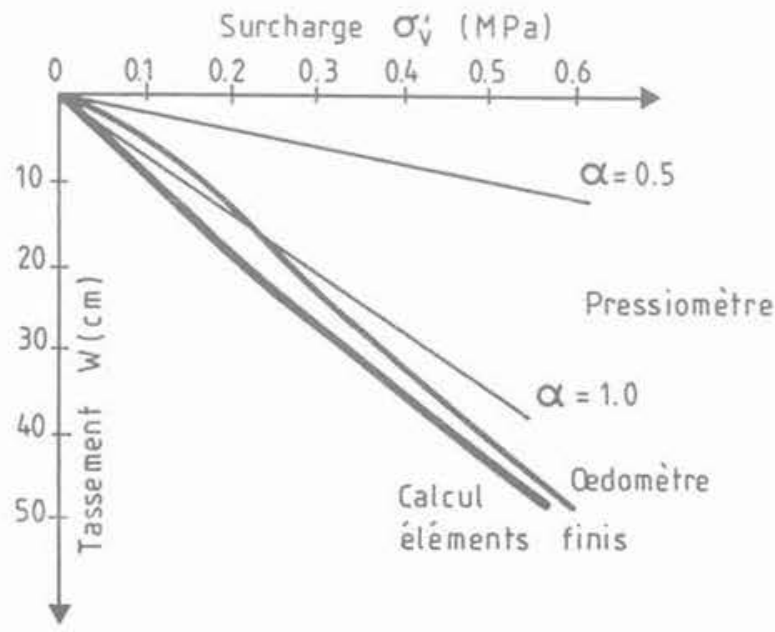

Fig. 13. - Comparaison des tassements calculés par E.F. et prévus.

Fig. 13. - Comparison between finite element and forecasted settlements.

\section{DISCUSSION DES RÉSULTATS}

La comparaison des courbes de la figure 13 montre que le calcul œdométrique donne une valeur de tassements, au moins sous contrainte élevée (supérieure à $0,4 \mathrm{MPa}$ ), proche des valeurs obtenues par le calcul numérique. II convient de noter qu'en théorie, le tassement total sous la fondation résulte de la superposition du tassement instantané initial non drainé et du tassement de consolidation tel qu'on l'a évalué dans la méthode œedométrique, éventuellement corrigé d'un facteur multiplicatif $\mu$. Ce facteur tient compte de la différence de chemin de contrainte suivi par un élément de sol sous la fondation, pour lequel une déformation latérale est possible, et par l'échantillon œdométrique, SKEMPTON. BJERRUM [8]. L'application stricte de cette méthode, en retenant pour module de cisaillement celui pris en compte dans la loi de comportement et pour coefficient de Poisson une valeur 0,5 correspondant au chargement non drainé induit sous une charge de $0,5 \mathrm{MPa}$ de la fondation, un tassement instantané de $0,05 \mathrm{~m}$. Le facteur correcteur $\mu$ a été évalué à partir de la formule de Skempton :

$$
\mu=\mathrm{A}+(1-\mathrm{A}) \alpha
$$

où $\mathrm{A}=$ coefficient de Skempton relatif à la pression interstitielle

$\alpha=$ facteur dépendant de la géométrie du problème.

$\alpha=0,38$ pour une semelle circulaire reposant sur une fondation d'épaisseur égale à son diamètre.

D'après les résultats des essais triaxiaux réalisés sur l'argile du Gault inférieur, A est égal à 0,65 environ, ce qui conduit à une valeur de $\mu=0,78$. Le tassement cedométrique corrigé est alors de $0,32 \mathrm{~m}$ pour une charge de $0.5 \mathrm{MPa}$, ce qui ajouté au tassement instantané conduit à un tassement total de $0,37 \mathrm{~m}$. L'écart entre la valeur théorique de référence (calcul aux éléments finis) et cette valeur est inférieur à $15 \%$.

L'étude réalisée confirme donc les conclusions de BURLAND [4] : pour un sol ayant une réponse approximativement élastique sous chargement monotone (coefficient de sécurité vis-à-vis de la rupture supérieur à 2,5), le tassement total est correctement évalué par la méthode œdométrique. Des calculs plus élaborés faisant intervenir l'anisotropie ou séparant le phénomène instantané du phénomène à long terme ne donnent pas une meilleure évaluation.

L'interprétation classique de l'essai pressiométrique sous-estime les tassements. Cette interprétation nécessite la connaissance de deux facteurs : le coefficient rhéologique $\alpha$ et le coefficient de Poisson $\nu$ qui sont mal connus dans le cas des argiles surconsolidées. L'influence de coefficient de Poisson $\nu$ pris arbitrairement égal à 0,33 est faible, de l'ordre de $20 \%$. L'influence du coefficient $\alpha$ est nettement plus importante. Les calculs effectués montrent qu'il serait nécessaire de retenir une valeur de $\alpha>1$ (environ 1,1) pour retrouver les valeurs théoriques de référence. Rappelons que dans l'interprétation usuelle de l'essai pressiométrique, $\alpha$ est toujours inférieur ou égal à 1 . Ces résultats ne remettent pas nécessairement en cause le bien-fondé de l'approche pressiométrique mais ils attirent l'attention sur les limitations de la méthode: l'essai pressiométrique a été initialement 
développé pour des fondations de petites dimensions qui induisent dans le sol des états de contraintes essentiellement déviatoriques alors que les fondations de grands diamètres, comme celles considérées dans cette étude, induisent des états de contrainte principalement sphériques ; la valeur du coefficient rhéologique $\alpha$ n'a pas été étalonné sur un grand nombre de sites constitués d'argile fortement surconsolidée.

\section{CONCLUSIONS}

L'étude montre que pour une fondation de grand diamètre reposant sur une argile fortement surconsolidée, la méthode de calcul du tassement à partir d'essais œdométriques est fiable. Bien entendu, l'évaluation n'est correcte que si le prélèvement et l'essai lui-même sont de bonne qualité. On a vu, en particulier, qu'un prélèvement même parfait, pouvait conduire à une détente de l'échantillon du fait de la compressibilité du fluide interstitiel. Cette décompression a pour effet de décaler la courbe œedométrique et de diminuer la valeur de la contrainte pour laquelle le tassement débute. Pour s'affranchir de ce phénomène, il convient bien de calculer le tassement à partir de la contrainte correspondant au poids des terres et non à partir de cette pseudo-pression de consolidation. Le calcul à partir de l'essai pressiométrique reste entièrement tributaire de la valeur du coefficient rhéologique $\alpha$. L'étude a montré que pour le matériau étudié, une valeur de $\alpha$ légèrement supérieure à 1 , et donc en dehors des règles pressiométriques, serait nécessaire pour obtenir une valeur égale à celle du tassement théorique. Néanmoins la valeur 1 , qui résulterait de l'application des normes pressiométriques en ne tenant pas compte de la très faible valeur du rapport $\mathrm{E} /\left(\mathrm{p}_{1}-\mathrm{p}_{0}\right)$, donne un tassement convenable, bien qu'inférieur à la simulation numérique pour les fortes charges. La valeur de $\alpha$ pour l'argile du Gault apparaît en dehors des normes. Il resterait à préciser cette valeur de $\alpha$ pour d'autres matériaux et à imaginer une méthode de mesure de $\alpha$ à partir de l'essai pressiométrique.

Finalement, les calculs présentés ont montré qu'on disposait d'un outil numérique efficace. Cet outil pourrait permettre de simuler des essais pressiométriques sur d'autres sols surconsolidés afin de préciser la valeur de $\alpha$ à prendre en compte pour permettre le calcul du tassement à partir de l'essai pressiométrique mêrme pour des fondations aussi exceptionnelles que celle étudiée.

De façon plus ambitieuse, on peut envisager d'évaluer à l'avenir les tassements d'ouvrages importants par des calculs en éléments finis, en utilisant une loi de comportement représentative du comportement réel du matériau, correctement calée et validée sur les résultats d'essais tant in situ que de laboratoire. Ces essais devraient être effectués sur un petit nombre d'échantillons très soigneusement prélevés et testés, COSTAZ, HAGHGOU [5], BOZETTO, OLLAGNIER [3], BIAREZ et al [2].

\section{BIBLIOGRAPHIE}

[1] BAGUELIN F., JEZEQUEL J.F., SCHIELDS D.H. (1978), "The pressuremeter and foundation engineering $*$. Transtech Publications.

[2] BIAREZ J. et al (1989), "Interprétation de l'essai pressiométrique pour la prévision des tassements *, Communication au Congrès de Rio, à paraître.

[3] BOZETTO P., OLLAGNIER M. (1986), * La mécanique des sols au SEPTENः, Revue des laboratoires d'essais.

[4] BURLAND J.B., BROWNES B.D., MEL. LO V.F.B. (1977), «Behaviour of foundation and structure ». Proceedings of the 9th International Conference on Soil Mechanics and Foundation Engineering, Tokyo.

[5] COSTAZ J.L., HAGHGOU M. (1987), «Fondations des centrales nucléaires n, Revue française de Géotechnique n 41 .

[6] LUONG M.P. (1978), * Comportement cyclique des sols pulvérulents ", compte rendu Académie des Sciences, série B, t 287.

[7] PRÉVOST J.H. (1985), *A simple plasticity theory for frictional cohesionless soils », Journal of Soil Dynamic and Earthquake Engineering, vol. $4, n^{\circ} 1$.

[8] SKEMPTON A.W., BJERRUM L. (1987), *A contribution to the settlement analysis of founda. tion on clay », Géotechnique, vol. 7, n 4, 1957. 